\title{
Discussion on the Mechanism of Action of High-frequency Drugs for Treating Heart Failure Based on Network Pharmacology
}

\author{
Feifei Lei ${ }^{1}$, Mingjun Zhao ${ }^{1}{ }^{2}$, Haifang Wang ${ }^{1}$, Chao Pan ${ }^{1}$, Xiaoya Shi $^{1}$ \\ ${ }^{1}$ Shaanxi University of Traditional Chinese Medicine, Xianyang 712046, Shaanxi Province, China \\ ${ }^{2}$ Affiliated Hospital of Shaanxi University of Traditional Chinese Medicine, Xianyang 712000, Shaanxi Province, China
}

Funding: Project of Establishment of the Famous Traditional Chinese Medicine Doctor Zhao Mingjun Studio (No.2019007).

\begin{abstract}
[Abstract] Objective: To explore the target and mechanism of Astragalus membranaceus, poria, salvia miltiorrhiza and semen leiocarpa in the treatment of heart failure by network pharmacology. Methods: The active components of traditional Chinese medicine and the target of heart failure were screened by multi-platform, and the standard gene was transformed by Uniprot. CytoCasp 3.6.1 was used to draw the network diagram of traditional Chinese medicine - component - target. Go and KEGG analysis were performed by Metascape. Results: A total of 36 predictive target sites of Radix Astragalus, Fuling poria, Salvia miltiorrhiza and Draba nemorosa were screened for treatment of heart failure, mainly involving nerve and factor pathways: ADRB2, ADRA1B and AChE. Cancer pathway: TP53, TNF; Pathways of inflammation: IL1B, PTSG2, PTSG1; Sex hormone pathway: ESR1, AR, PGR; Others: SCN5A, HIF1A, etc. The results of GO and KEGG enrichment suggested that the treatment of heart failure with the top four drugs involved cancer pathway, calcium signaling pathway, HIF-1 signaling pathway, and involved in blood circulation, cell proliferation and other processes. Conclusion: This study combines the pharmacological studies of Chinese medicine and western medicine to reveal the mechanism of multi-target and multi-channel regulation of body balance in Chinese medicine treatment.
\end{abstract}

Key words: Network pharmacology; Chronic heart failure

Publication date: May, 2021; Publication online: 31 May, 2021

*Corresponding author: Mingjun Zhao, zmj7125@163.com

Heart failure is the traditional Chinese medicine "water disease", "heart water" that is, the pathogenesis for heart qi promotion, warming is not good, resulting in qi deficiency, blood stasis, edema. Chronic heart failure is often seen in the elderly viscera qi failure, qi deficiency and blood circulation water weakness into chronic heart failure qi deficiency and blood stasis water stop syndrome. Based on previous data mining results, this study suggests that the top four drugs in TCM treatment of heart failure are Astragalus membranaceus, poria, salvia membranaceus and semen leiocarpa var. leiocarpa. Therefore, network pharmacology method is used to study the pharmacological mechanism of the four high-frequency drugs in the treatment of heart failure, in order to provide a new direction for the research and development of Chinese medicine.

\section{Network pharmacology}

Since different compatibility of traditional Chinese medicine can treat various diseases, it is speculated that traditional Chinese medicine and compound medicine have the characteristics of multi-component and multi-channel regulation, so the platform of traditional Chinese medicine composition is used to search its chemical composition and explore the mechanism, which is a new idea for the current 
research of traditional Chinese medicine.

\section{Network pharmacological research}

\subsection{Drug composition screening and target collection}

(1) Apply TCMSP ( $\mathrm{OB}(\%)>30$, DL $>0.18)$, BATMAN platform ( Score cutoff $\geq 20, \mathrm{P} \geq 0.05$ ), TCMSP $(\mathrm{OB}(\%)>30$, $\mathrm{DL}>0.18$ ), to screen interacting protein by respectively inputting astragalus, tuckahoe, the root of red-rooted salvia, semen lepidii. Uniprot platform was used to name the standard genes of the proteins.

(2) Use Genecards and OMIM disease databases to collect heart failure targets and remove duplicates.

(3) The Venn platform was used to intersect the component targets and disease targets to obtain the target target of the four medicines for the treatment of heart failure.

2.2 Import the file into Cytoscape software to obtain the visualization of TCM components and targets, and adjust parameters to highlight

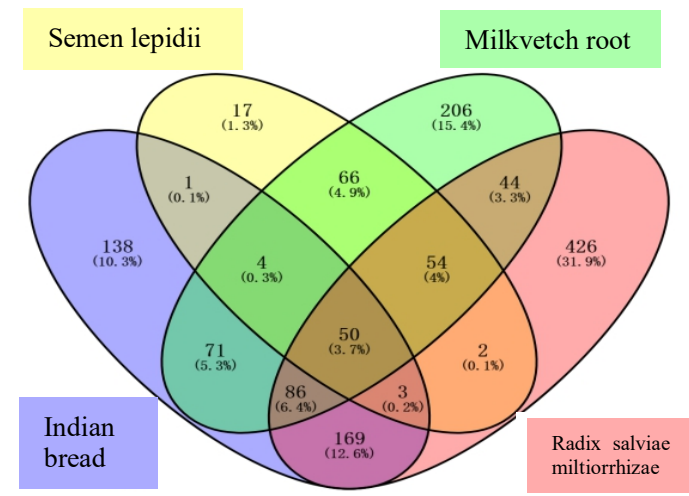

Figure 1. Intersection diagram of four drug targets

\section{important targets.}

2.3 Key targets were input into Metascape for ontology function analysis and enrichment pathway analysis.

\section{Conclusion}

\subsection{Target gene collection}

The active ingredients and targets of the four drugs were obtained through TCMSP and Batman database, and 176 active ingredients and 1445 targets were obtained after combining and de-weighting.

A total of 3456 Heart failure targets were obtained after Gene Cards (Score $\geq 7.655$ ) and OMIM disease database were searched for "Heart failure" combined with deduplication.

Thirty-six common targets of four herbs of milkvetch root, Indian bread, Semen lepidii and Radix salviae miltiorrhizae in the treatment of heart failure were obtained by using Venn diagram (Figure 1, Figure 2).

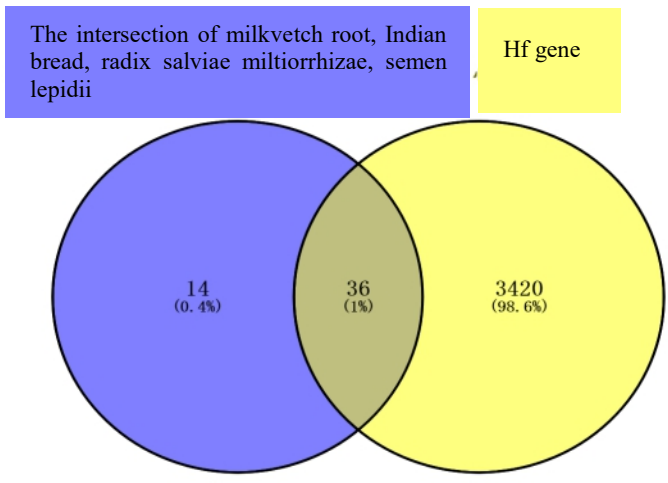

Figure 2. Intersection diagram of the intersection of four drugs and heart failure targets

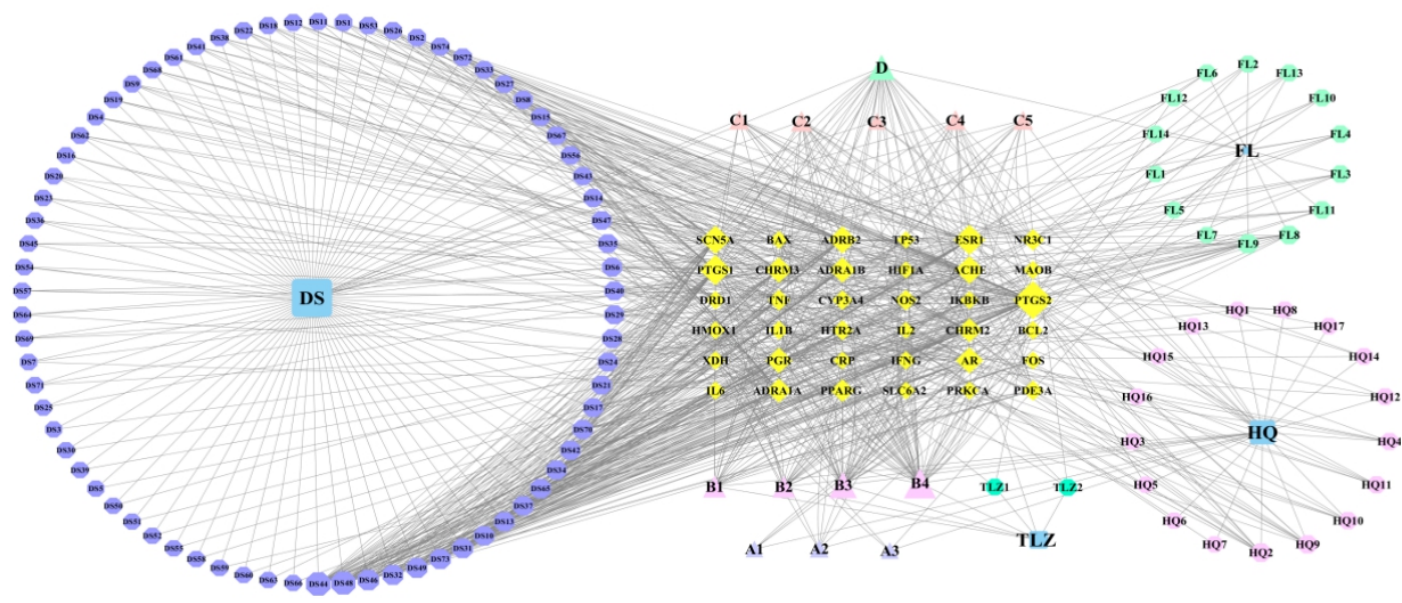

Figure 3. Chinese medicine - composition - target network diagram 


\subsection{GO functional analysis}

The cell composition mainly affects the membrane raft and the components of the presynaptic membrane (Figure 3). Molecular function mainly involves the G-protein-coupled amine receptor activity and nuclear receptor activity, etc. A histogram of $\mathrm{BP}, \mathrm{CC}$ and $\mathrm{MF}$ results was drawn based on the $P$ value (Figure 4).

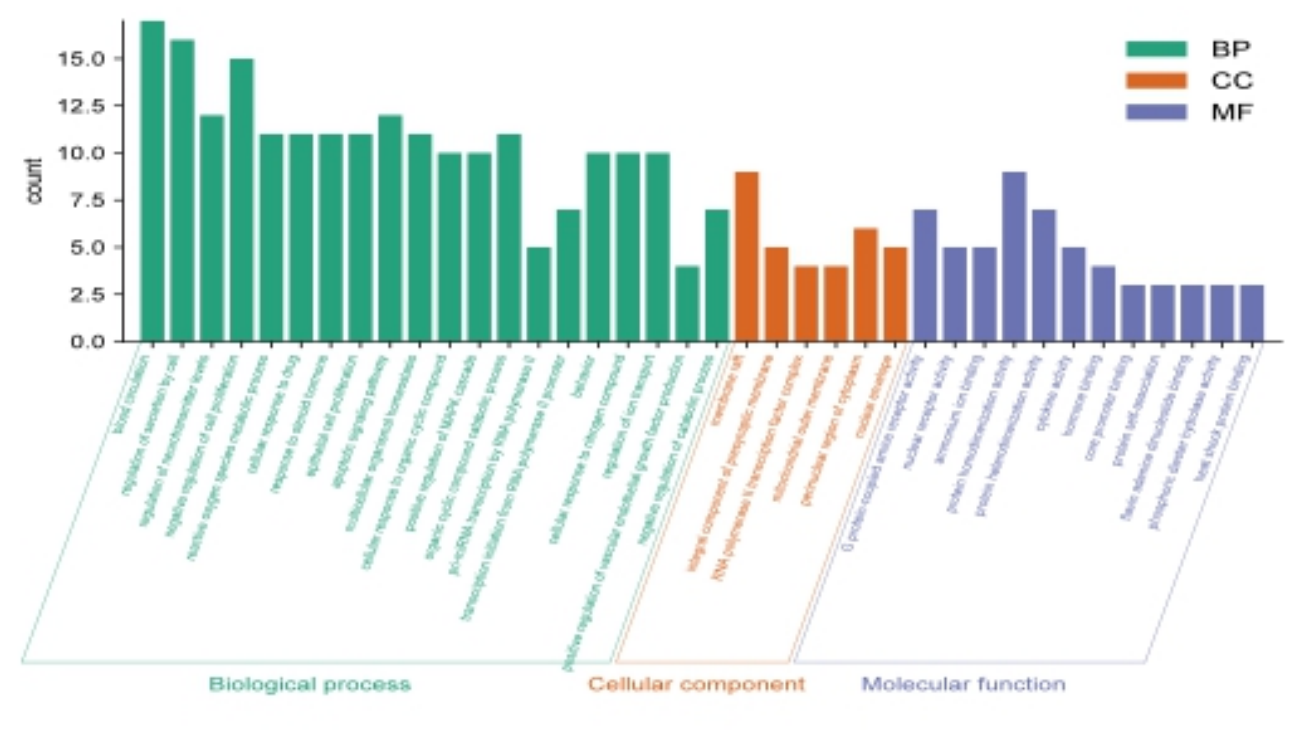

Figure 4. GO figure

\subsection{KEGG enrichment}

Signals mainly covered cancer pathways, HIF-1 pathway, calcium pathway, etc. Signal pathways were plotted as pathway bubble diagram according to the order of $\mathrm{P}$ value ( Figure 5 ).

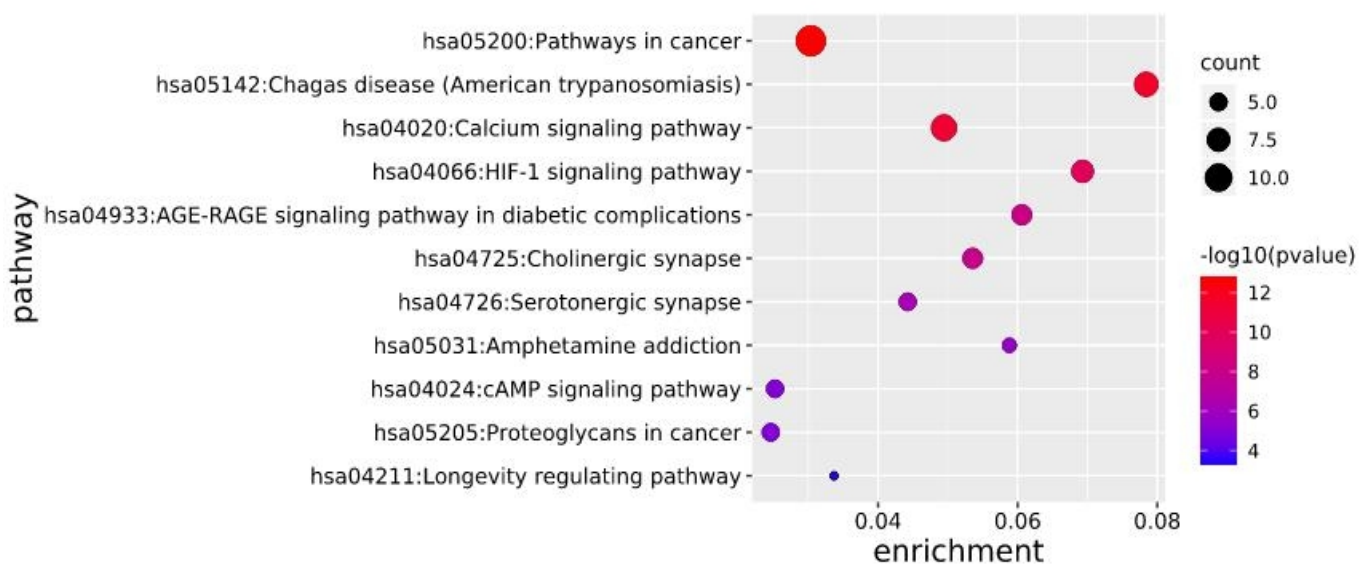

Figure 5. KEGG enrichment diagram

\section{Discussion}

Astragalus membranaceus mainly consists of astragalus saponins, flavonoids, polysaccharides, etc ${ }^{[1]}$. And it has diuretic, cardiostatic, anti-hypoxia and vasodilation effects ${ }^{[2]}$. Animal experiments showed that poria cocos could inhibit the expression of AQP2 and increase urine excretion. Draba nemorosa can increase cardiac output, reduce anterior and posterior load, and has no digitalis accumulation toxicity ${ }^{[3]}$.
It can inhibit the RAAS system and inhibit ventricular remodeling ${ }^{[4]}$. Salvia miltiorrhiza can inhibit RASS metabolism and regulate catecholamine to prevent myocardial remodeling. Tanshinone II A expanding coronary artery and reduce myocardial oxygen consumption ${ }^{[5]}$.

Through network pharmacological analysis, the key targets of Astragalus membranaceus, Salvia miltiorrhiza, Fulia and Draba nemorosa in the treatment of heart failure are ADRB2, ADRA1B, TNF $\alpha$, FOS, PTGS, SCN5a, ESR1 
and so on. ADRB2 belongs to the sympathetic nervous system and affects the cardiovascular system by regulating water and salt metabolism. Overexpression of ADRA1B reduces systolic response to $\beta$-AR activation in heart failure $^{[6]}$. FOS is related to cell differentiation and apoptosis. Astragalus polysaccharide and tanshinone can inhibit myocardial NF- $\kappa \mathrm{B}$ activity, reduce TNF- $\alpha$ and IL-6 levels, and improve cardiac function grading ${ }^{[7]}$. Studies have shown that the levels of CA125 and CA19-9 in heart failure patients are significantly higher than those in non-heart failure patients $^{[8]}$. In addition, the use of PTGS1 and PTGS2 inhibitors also increases the risk of $\mathrm{HF}^{[9]}$.

Studies on the relationship between sex hormones and heart failure have shown that the ratio of $\mathrm{T} / \mathrm{E} 2$ is related to the development of HF events ${ }^{[10]}$. SCN5A encodes cardiac sodium channels, and strict regulation of this gene ensures a stable heart rhythm ${ }^{[11]}$

GO and KEGG enrichment results showed that astragalus membranaceus, salvia miltiorrhiza, poria and semen leiocarpa involved cancer pathway, calcium signal, HIF-1 signal, cAMP signal and other pathways in the treatment of heart failure, and participated in blood circulation, cell secretion, cell proliferation and other biological processes. HIF1A induces VEGF expression, promotes angiogenesis and prevents myocardial hypoxia, which is an important therapeutic direction for heart failure ${ }^{[12]}$. CAMP is involved in cardiac excitation and renal water retention, etc., and the lower the cAMP content, the more serious the heart failure ${ }^{[13]}$. Calcium signaling pathway regulates myocardial contractibility, and the study of a new $\mathrm{Ca}^{2+}$ release channel stabilizer is a new direction for the treatment of heart failure ${ }^{[14]}$.

In conclusion, network pharmacology showed that astragalus membranaceus, poria cocos, salvia miltiorrhiza, semen lepidii key targets for treatment of heart failure on nerve factor, inflammation, cancer, and ion sources, etc., and GO and enrichment of KEGG pathway analysis are consistent with heart failure mechanism, biological process, explains the use of pharmacology method can provide methods for TCM modernization research, contribute to the vigorous development of traditional Chinese medicine in the future.

\section{References}

[1] Liu PN. Effect of Astragalus membranaceus decoction combined with western medicine on the treatment of chronic heart failure $[\mathrm{J}]$. Electronic Journal of Cardiovascular Diseases of Integrated Traditional and Western Medicine, 2017, 5(30): 187.

[2] Shi L, Ye W, Liu GG. Analysis of the application of traditional Chinese medicine in patients with chronic heart failure [J]. Chinese Journal of Medical Sciences, 2020, 10(14): 47-50 (in Chinese with English abstract).

[3] Zhou SP. Draba nemorosa var. leiocarpa and Draba nemorosa var. leiocarpa in the treatment of heart failure [J]. Chinese Journal of Traditional Chinese Medicine Emergency, 2014, 23(2): 271+314 (in Chinese with English abstract).

[4] Guo J, Chen CX, Shen YH. Effects of semen semen semen semen leiocarpa var.leiocarpa on experimental ventricular remodeling in animals $[\mathrm{J}]$. Chinese Journal of Traditional and Herbal Medicine, 2007(10): 1519-1523.

[5] Liu F, Zheng Z. Effects of Salvia miltiorrhiza on left ventricular hypertrophy in spontaneously hypertensive rats [J]. Chinese Journal of Emergency Medicine, 2003(11): 21-23.

[6] Woodcock EA. Roles of alpha1A- and alpha1B-adrenoceptors in heart: insights from studies of genetically modified mice [J]. Clin Exp Pharmacol Physiol. 2007 Sep;34(9):884-8. doi: 10.1111/j.1440-1681.2007.04707.x. PMID: 17645635.

[7] Yang ZX, Li ZB, Lin Q, et al. Effects of Astragalus polysaccharide combined with tanshinone on $\mathrm{NF}-\kappa \mathrm{B}$ activation of MIF, TNF- $\alpha$ and IL-6 expression in rats with chronic heart failure $[\mathrm{J}]$. Chinese Journal of Traditional Chinese Medicine, 2012,27(12): 3221-3225.

[8] Shi C, van der Wal HH, Silljé HHW, et al.Tumour biomarkers: association with heart failure outcomes $[\mathrm{J}] . \quad \mathrm{J}$ Intern Med.2020 Aug; 288(2):207-218.doi: 10.1111/joim.13053.Epub 2020 May 5. PMID: 32372544; PMCID: PMC7496322.

[9] Zhu L, $\mathrm{Xu} \mathrm{C}$, Huo $\mathrm{X}$, et al. The cyclooxygenase-1/mPGES-1/endothelial prostaglandin EP4 receptor pathway constrains myocardial ischemia-reperfusion injury. Nat Commun. 2019 Apr 23;10(1):1888. doi: 10.1038/s41467-019-09492-4. PMID: 31015404; PMCID: PMC6478873.

[10] Zhao D, Guallar E, Ouyang P, et al. Michos ED. Endogenous Sex Hormones and Incident 
Cardiovascular Disease in Post-Menopausal Women [J]. J Am Coll Cardiol.2018 Jun 5;71(22): 2555-2566.doi: 10.1016/j.jacc.2018.01.083.PMID: 29852978; PMCID: PMC5986086.

[11] Shang LL, Pfahnl AE, Sanyal S, et al. Human heart failure is associated with abnormal C-terminal splicing variants in the cardiac sodium channel. Circ Res.2007 Nov 26;101(11):1146-54.doi: 10.1161/CIRCRESAHA.107.152918.Epub 2007 Sep 27.PMID: 17901361; PMCID: PMC3157752.

[12] Shi XJ, Wei YH, Tang JY. Research advance of HIF-1 $\alpha$ in the treatment of heart failure with traditional Chinese medicine $[\mathrm{J}]$. Chinese Journal of Traditional Chinese Medicine,2020,38(01):118-121.

[13] Li ZK, Zhu SJ, Tian Y et al. Antimicrobial pathway analysis of heart failure in patients with heart failure [J]. Chin J Cardiol,2010, 2(4): 242-246 (in Chinese).

[14] Chen P, Guo LH, Guo YK, et al. dentification of disturbed pathways in heart failure based on Gibbs sampling and pathway enrichment analysis[J]. Enet Mol Res.2016 Apr 28;15(2).doi: 10.4238/gmr.15027956. PMID: 27173293. 